\title{
Saliva Production Inhibition
}

National Cancer Institute

\section{Source}

National Cancer Institute. Saliva Production Inhibition. NCI Thesaurus. Code C41483.

Saliva Production Inhibition involves interference with, or restraint of, synthesis of the clear, slightly acid (pH 6.8) viscous fluid secreted from the parotid, sublingual, and submandibular salivary glands and the mucous glands of the oral cavity that contain mucins, water, organic salts, and ptylin and keep the oral mucous membrane moist, lubricate food, and partially convert starch into maltose. 